\title{
Deaths from rare protozoan encephalitis in Karachi blamed on unchlorinated water
}

Owing to a coding error, the names of this News story's two authors (Abdul Nafey Kazi and Talha Riaz) have appeared initially as one name in PubMed, despite appearing correctly 